\title{
Research on Optimization of Delivery Route of Online Orders
}

\author{
Zhao Qingju \\ School of Information Beijing Wuzi University, No.321, Fuhe Street, Tongzhou District, Beijing 101149, P.R.China \\ Email: $776335520 @ q q . c o m$
}

\begin{abstract}
With the popularity of the Internet, the accelerating of the rhythm of people's life, ordering food online has become a new way of life. Under the background of ordering food on the Internet, how to arrange distribution routes and deliver meals to customers in time has become an urgent problem to be solved after the customer's order. The "first come first served" mode of traditional catering reduces the efficiency of orders and increases the operation cost of businesses. Sometimes, due to the shortage of distribution staff, the order waiting time increases. As a result, order delays and customer satisfaction are reduced. The reputation of the restaurant has also been affected. This paper starts from solving practical problems, considering customer location and customer needs, and tries to build two stage algorithm to optimize order routing problem. First, we use the idea of "clustering path optimization" to aggregate customers to $\mathrm{K}$ areas according to the location of our customers. On this basis, the capacity limit of distribution vehicles is increased. This will make the customer point in the region closer. And save operation cost. Then, the genetic algorithm is used to optimize the delivery path in the K class area. Finally, the practicability and effectiveness of the algorithm are verified by simulation experiments.
\end{abstract}

Keywords: Online ordering; regional division; K-means clustering; genetic algorithm

\section{Introduction}

In recent years, with the popularity of the Internet, people's pace of life is accelerating. Due to its unique convenience and intuition, online ordering has become a new way of life for many office workers and students. According to the data, the market size of China's catering industry in 2016 was 3.6 trillion yuan, with an annual growth rate of $10.8 \%$. It is estimated that by 2020, the market size can exceed 5 trillion yuan. On August 24, 2017, Trustdata released the "Analysis Report on the Development of China's Take-out Industry in the First Half of $2017^{\text {"[1] }}$, which pointed out that the amount of take-away transactions in the first half of 2017 was nearly 100 billion, and the MeiTuan take-out and ELeMe accounted for more than $80 \%$. As of the end of June 2017, the number of take-away users has doubled year-on-year, and DAU has nearly 15 million. The latest "2016 China Takeaway O2O Industry Development Report" published by iResearch ${ }^{[2]}$ shows that the scale of China's food and beverage outlet market in 2015 has exceeded 230 billion yuan, accounting for $7.4 \%$ of the overall food and beverage consumption. By 2018 this ratio is expected to reach 14.8\%. The overall size of the take-away market will exceed 660 billion yuan, the market size of the take-out $\mathrm{O} 2 \mathrm{O}$ market is 44.24 billion yuan, and the penetration rate in the take-away market is $18.5 \%$. It is expected that the market for take-away O2O will exceed 150 billion yuan in 2018. The above data reflects the huge potential of the online ordering market. The overall size of the takeaway market will also exceed 660 billion. The above data reflect the huge potential of the online ordering market.

While online ordering is developing at a rapid pace, it is also facing the common problem of delay in delivery. Sootoo Research Institute "February 2015 catering O2O market survey report" [3] shows that, in the catering $\mathrm{O} 2 \mathrm{O}$ takeaway complaint, the slow delivery rate was $63 \%$, and the food safety was $27 \%$. It can be seen that the delay in delivery of goods has become the main bottleneck that hinders the expansion of the online ordering market.

This paper starts from solving practical problems, considering customer location and customer needs, and tries to build two stage algorithm to optimize order routing problem. First, we use the idea of "clustering path optimization" to aggregate customers to $\mathrm{K}$ areas according to the location of our customers. On this basis, the capacity limit of distribution vehicles is increased. This will make the customer point in the region closer. And save operation cost. Then, the genetic algorithm is used to optimize the delivery path in the K class area. Finally, the practicability and effectiveness of the algorithm 
are verified by simulation experiments.

\section{Problem Description}

This paper studies the optimization of the distribution path of the online ordering platform. It consists of a distribution center and multiple customers. Due to the high timeliness of takeaway, the first-come-first-delivery mode of traditional catering reduces the efficiency of orders and increases the operation cost of businesses. Sometimes, due to the shortage of distribution staff, the order waiting time increases. As a result, order delays and customer satisfaction are reduced. The reputation of the restaurant has also been affected. This paper comprehensively considers the customer location and customer demand, starting from solving the actual problem, trying to construct a two-stage algorithm to optimize the order routing path problem. The key decision issues include: 1) How to carry out reasonable customer division, save operating costs, reduce the no-load rate of distribution vehicles, reducing the invalid route of the delivery vehicle.2) How to design a reasonable distribution order for the divided areas, that is, optimize the distribution path.

At present, the main methods of distribution area division are scanning method ${ }^{[4]}$ and clustering algorithm ${ }^{[5]}$. The basic idea of scanning method is to solve the problem of logistics distribution vehicle scheduling by successive approximation. Generally applicable to a small number of customers, the distribution area is not much. When the number of customer outlets is large and there are many distribution areas, the clustering algorithm is adopted. A large number of d-dimensional data objects (n) are aggregated into $\mathrm{k}$ clusters $(\mathrm{k}<\mathrm{n})$, so that the similarity of data objects in the same cluster is maximized, and the similarity of objects in different clusters is minimized. Due to the particularity of clustering, in the logistics system, the use of clustering algorithm to divide the distribution area can make the area to be closer and more in line with actual needs. When clustering, there are mainly the following aspects to consider: First, the number of clusters. This is a value that needs to be entered first, which must consider the actual situation and should be calculated based on the total daily delivery volume and bicycle capacity. Because the customer's needs are different in each cycle, the value needs to be recalculated at the beginning of each delivery cycle ${ }^{[6]}$. Second, the points within each cluster should be relative concentration, so that the vehicle does not spend a lot of time on the road. Third, the workload of each cluster should be roughly the same. In this way, it can avoid the situation that a delivery vehicle has a particularly small or a lot of delivery. Fourth, every two clusters cannot overlap, otherwise repeated delivery will occur; similarly, each point must be included in a certain class ${ }^{[7]}$. Fifth, in practical applications, only the distribution of customers located in the urban area is considered. For customers located in the suburbs, the distribution principle of the urban area can be used for calculation.

\section{Two-stage Distribution Path Optimization Algorithm}

The division of logistics distribution area is a combination optimization problem, it is a multi-constrained and multi-objective decision-making optimization problem, which belongs to NP-hard problem ${ }^{[8]}$. The current time complexity of the general solution to VRP problem is mostly $\mathrm{O}(\mathrm{n} 2)$. If this method is used to calculate the vehicle path problem with a scale of about 10,000 nodes, it is expected to take at least several hundred hours. Therefore, it is necessary to find a suitable solution to solve the problem of large-scale vehicle routing. The current solution is to turn it into zero and break it down. Firstly, according to certain constraints and using the corresponding methods, the large-scale complex distribution network is divided into several small-scale distribution areas. Then solve the small-scale vehicle routing problem one by one. Design the optimal distribution route in each sub-area to find the approximate problem of the whole problem.

The idea of two-stage heuristic algorithm is that the order is usually distributed by region in the e-commerce environment, and each delivery area is responsible for one car. This paper is based on the idea of "cluster-path optimization" [9], First use k-means clustering method to cluster customers, and then uses the genetic algorithm to optimize the distribution routes of each region to minimize the delivery time. The overall flowchart of the algorithm is as follows: 


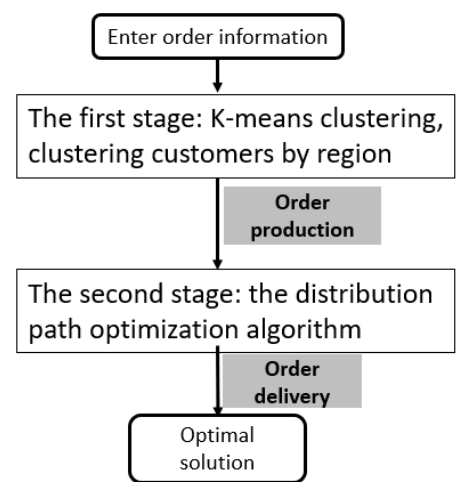

Figure 1. Algorithm overall flow chart

\subsection{The First Stage: K-Means Clustering, Clustering Customers by Region}

The K-means clustering method is used to classify customers into $\mathrm{V}$ categories, $N=\left\{\sigma_{1}, \sigma_{2} \ldots, \sigma_{v}, \ldots, \sigma_{V}\right\}$, and the order capacity of each type of customer set $\sigma_{v}$ does not exceed the maximum capacity $\mathrm{Q}_{\mathrm{v}}$ of the vehicle, and the customer clusters by region. The basic flow chart is as follows:

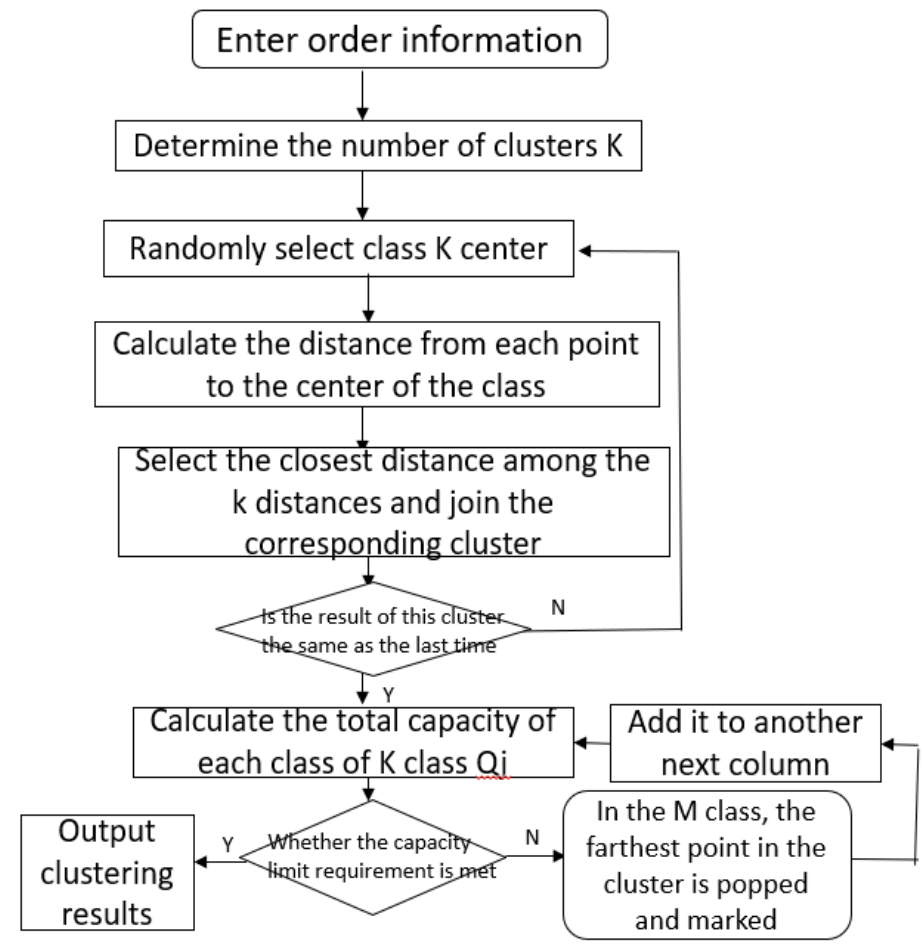

Figure 2. Basic flow chart of customer clustering by region

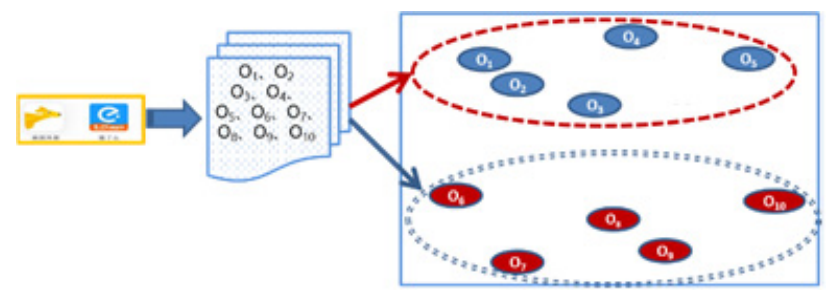

Figure 3. Customer clustering by region 
The specific steps of the K-means algorithm are summarized as follows:

Step 1: According to the number of delivery staff and the capacity of the delivery box, the volume of the food packaging box is calculated, and the customers are grouped into $\mathrm{k}$ categories.

Step 2: Select k initial nodes as the cluster center.

Step 3: Calculate the class that each customer point should belong to, to meet the minimum distance from each customer to the center of mass: $c^{(i)}=\arg \min _{j}\left\|x^{(i)}-\sigma_{j}\right\|^{2}, \quad \sigma_{j}=\left\{o_{1}, o_{2}, \cdots, o_{i}\right\}, i \in n$.

Step 4: Clustering by K-means algorithm to obtain k clusters.

Step 5: Calculate the sum $Q_{j}$ of each class of customer's demand.

Step 6: Compare the sum of the demand of each type with the size of the car capacity , to determine whether it exceeds the maximum capacity of the type of delivery box: $\sum_{i=1}^{n} \sigma_{j}\left(O\left(q_{i}\right)\right) \leq Q_{V}, j \in V$.

Step 7: If $\sum_{i=1}^{n} \sigma_{j}\left(O\left(q_{i}\right)\right)>Q_{V}, j \in V$, in the M class, the farthest point of the cluster is popped up and marked, and this tag is not processed in future loop iterations. Turn to the Sub distance point. Add this point to the class closest to $\mathrm{m}$, and recalculate $\mathrm{Q}_{\mathrm{j}}$. Go to Step 6 and continue checking until it converges.

\subsection{The Second Stage: Distribution Path Optimization}

The traditional genetic algorithm is used to optimize the path of each type of customer, which can be transformed into the traditional traveling salesman problem (TSP). Detailed genetic algorithm steps are not described again. The general flow chart of the genetic algorithm in this paper is as follows:

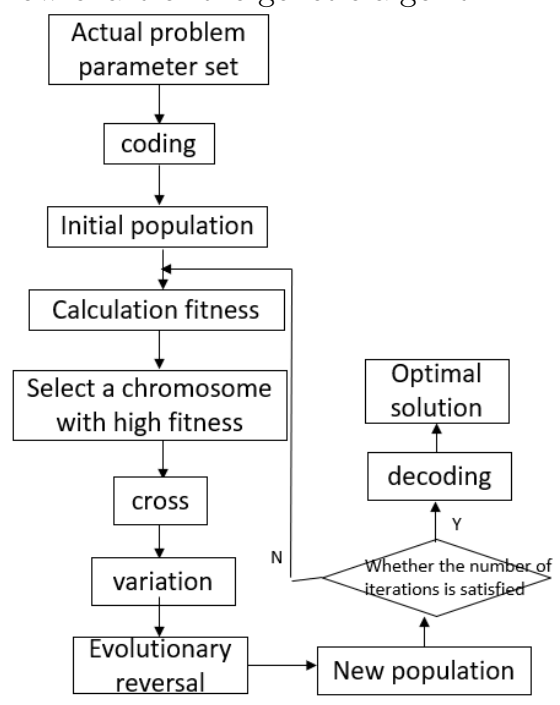

Figure 4. Flow chart of solving the TSP problem of genetic algorithm

Genetic algorithm rough steps:

Step 1: Encoding: Encoding method using integer arrangement.

Step 2: Population initialization: After completing the chromosome coding, an initial population must be generated as the starting solution. So first you need to decide the number of initialized populations. Its value floats between 50 and 200 .

Step 3: Fitness function: The fitness function takes the reciprocal of the delivery time. The goal of optimization is to select the chromosome with the largest fitness function value. The higher the fitness function value, the better the quality of the chromosome, and the worse the quality.

Step 4: Select operation: Select the operation to select individuals from the old group with a certain probability to the new group. The probability that an individual is selected is related to the fitness value. The greater the individual fitness value, the greater the probability of being selected.

Step 5: Crossover: Partial mapping hybridization.

Step 6: Mutation operation: The mutation strategy takes two points randomly.

Step 7: Evolutionary reversal operation: In order to improve the local search ability of the genetic 
algorithm, after multiple choices, crossovers, and mutations, successive evolutionary reversal operations are introduced.

\section{Case Analysis}

In the actual dispatch, the data should be obtained according to the customer's demand characteristics under the online ordering situation. Therefore, in order to verify the validity of the model and algorithm, this paper constructs an example based on the actual situation of an online ordering merchant. The algorithm is implemented with MatlabR2017 b. The computer hardware configuration is: operating system Windows 7 Ultimate, memory 2.00GB; processor Intel (R) Core (TM) 2 Duo 3.00GHz.

According to the hypothesis and actual investigation, the relevant parameters are set as follows: Assume that within a certain period of time, the online ordering enterprise receives an order from $n=40$ customers in a certain area, the serial number 0 indicates the distribution center, the serial number $\{1,2, \ldots, 40\}$ indicates 40 customer points. At this time, the delivery vehicle of the same model is in an idle state, and the delivery vehicle speed is $\mathrm{V}=20 \mathrm{~km} / \mathrm{h}$. The number of delivery vehicle $\mathrm{k}=8$, and the maximum capacity of the delivery vehicle is $Q=12$, the business coordinates are $(0,0)$. The coordinates and demand of each customer point are shown in Table 1. The time window for the order to be delivered to the customer is concentrated at the peak of the meal from 11:00 to 13:30. Assume that the customer's delivery window width is 30 minutes. To ensure the validity of the numerical experiment results, 10 experiments were repeated for each order environment, and the average was taken as the result of the comparison.

Table 1. Location coordinates and demand of each customer point

\begin{tabular}{cccccccc}
\hline Customer number & $\mathrm{X}$ & $\mathrm{Y}$ & Required quantity & Customer number & $\mathrm{X}$ & $\mathrm{Y}$ & Required quantity \\
\hline 1 & 1.7 & 0.8 & 3 & 21 & 0.6 & -1.0 & 1 \\
2 & 1.7 & 1.3 & 1 & 22 & 0.4 & -0.9 & 1 \\
3 & 0.7 & 1.9 & 2 & 23 & 0.7 & -2.0 & 2 \\
4 & 1.2 & 1.6 & 2 & 24 & 0.4 & -1.6 & 3 \\
5 & 1.7 & 1.0 & 2 & 25 & 1.0 & -1.0 & 2 \\
6 & 1.9 & 1.5 & 2 & 26 & 1.8 & -1.8 & 2 \\
7 & 1.3 & 0.8 & 4 & 27 & 1.3 & -0.3 & 3 \\
8 & 0.4 & 1.9 & 3 & 28 & 0.2 & -0.8 & 3 \\
9 & 1.3 & 2.0 & 3 & 29 & 0.8 & -1.9 & 3 \\
10 & 0.1 & 1.7 & 1 & 30 & 0.1 & -1.8 & 1 \\
11 & -1.0 & 1.7 & 2 & 31 & -0.3 & -2.0 & 2 \\
12 & -1.9 & 1.2 & 3 & 32 & -1.2 & -0.6 & 4 \\
13 & -1.4 & 1.9 & 1 & 33 & -1.4 & -1.9 & 3 \\
14 & -0.6 & 0.1 & 2 & 34 & -0.8 & -1.4 & 1 \\
15 & -0.6 & 1.2 & 3 & 35 & -1.9 & -1.0 & 3 \\
16 & -1.7 & 0.6 & 3 & 36 & -1.9 & -1.3 & 2 \\
17 & -1.8 & 1.7 & 2 & 37 & -1.3 & -1.8 & 3 \\
18 & -1.3 & 0.4 & 3 & 38 & -1.7 & -0.4 & 2 \\
19 & -0.5 & 0.9 & 2 & 39 & -0.8 & -1.3 & 1 \\
20 & -0.2 & 0.8 & 1 & 40 & $-1.3-2.0$ & 2 \\
\hline
\end{tabular}

\section{Conclusion and Outlook}

The two-stage distribution path optimization algorithm proposed in this paper is compared with the traditional processing algorithm. The left side of Figure 5 is the clustering result graph of the k-means clustering algorithm. The right side of Figure 5 is a schematic map of the delivery path obtained by genetic algorithm. The results show that the two-stage distribution path optimization algorithm proposed in this paper can reduce the delivery delay time by about $29.81 \%$. The results show that the proposed two-stage 
allocation path optimization algorithm can effectively shorten the order delay time, reduce the delay penalty cost, improve customer satisfaction and save operating costs.
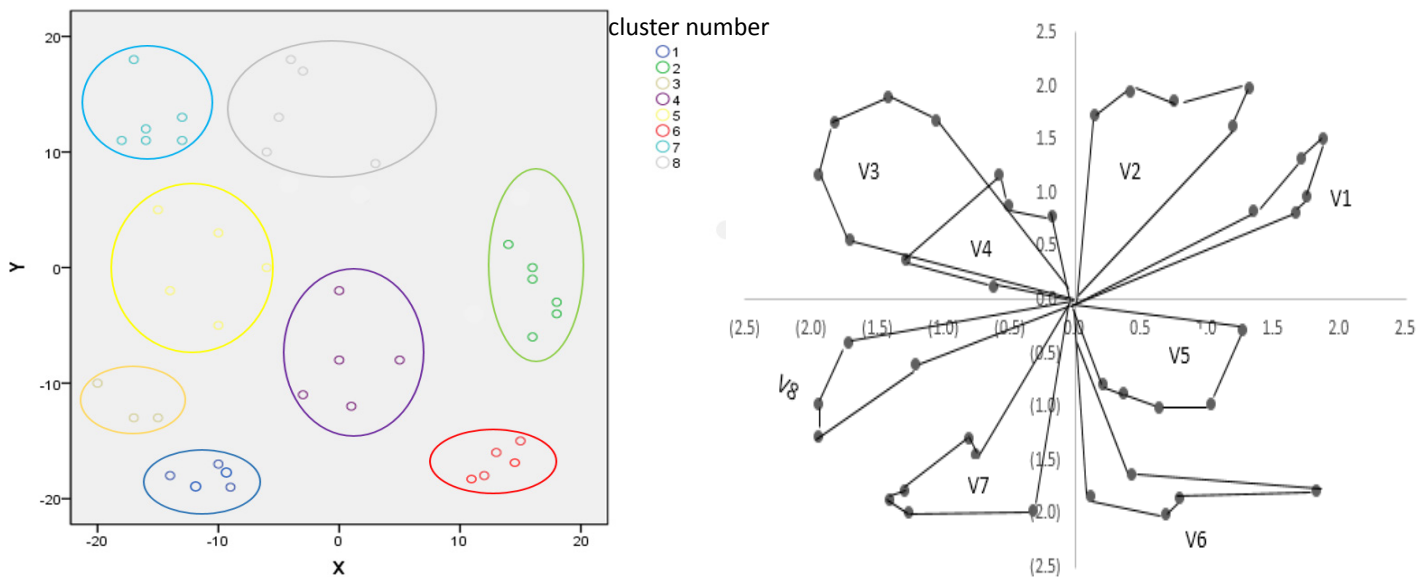

Figure 5. Schematic diagram of 40 customer distribution maps and distribution routes

This paper only considers the problem of order fulfillment within a single merchant under the predetermined mode. The next thing to study is the optimization of order delivery path for multiple businesses in real-time mode.

With the accelerated pace of life and the expansion of domestic demand proposed by the Chinese government, dining out and take-away meals will gradually become the dining habits of more and more users in China. In the future, we will have a safer food environment when the take-away companies continue to improve distribution efficiency and food safety management.

Project: Graduate Research and Innovation Quality Training Program (05417030301) [Beijing Intelligent Logistics System Collaborative Innovation Center]

\section{References}

1. Trustdata. Analysis report on the development of China's take-out industry in the first half of 2017 [EB/OL], 2017.8.24. http://www.itrustdata.cn/\#index

2. IResearch. 2016 China Outsourcing O2O Industry Development Report, [EB/OL], 2017. http://www.iresearch.com.cn/report/2615.html

3. Sootoo Research Institute. February 2015 Food and Beverage 020 Market Research Report[EB/OL],2015.3.12. http://www.sootoo.com/content/555675.shtml

4. Jianbin Li, Rihuan Huang \& James B. Dai.Joint optimisation of order batching and picker routing in the online retailer's warehouse in China [J].International Journal of Production Research, 2017, 55(2):447-461.

5. Hall N G, Potts C N. Supply chain scheduling: batching and delivery, [J]. Operations Research, 2003.51(4): 566-584.

6. Chen Z L, Vairaktarakis G L. Integrated scheduling of production and distribution Operations, [J]. Management Science Journal of the Institute for Operations Research\&the Management Sciences, 2005, 51(4): 614-628.

7. Pundoor G, Chen Z L. Scheduling a production-distribution system to optimize the trade off between delivery tardiness and total distribution cost, [J]. Naval Res. Logistic, 2005, 52: 571-89.

8. Garcia J. M., Lozano S., Smith K., et al. Coordinated scheduling of production and delivery from multiple plants and with time windows using genetic algorithms[J].Proceedings of the 9th International Conference on Neuronal Information Processing.2002,3: 1153-1158.

9. Ullrich C A. Integrated machine scheduling and vehicle routing with time windows, $[\mathrm{J}]$. European Journal of Operational Research, 2011, 227(1):152-165. 